\title{
ERF deletion rescues RAS deficiency in mouse embryonic stem cells
}

\author{
Cristina Mayor-Ruiz, ${ }^{1}$ Teresa Olbrich, ${ }^{1}$ Matthias Drosten, ${ }^{2}$ Emilio Lecona, ${ }^{1}$ Maria Vega-Sendino, ${ }^{1}$ \\ Sagrario Ortega, ${ }^{3}$ Orlando Dominguez, ${ }^{4}$ Mariano Barbacid, ${ }^{2}$ Sergio Ruiz, ${ }^{1}$ \\ and Oscar Fernandez-Capetillo, ${ }^{1,5}$ \\ ${ }^{1}$ Genomic Instability Group, ${ }^{2}$ Experimental Oncology Group, ${ }^{3}$ Transgenic Unit, ${ }^{4}$ Genomics Unit, Spanish National Cancer \\ Research Centre (CNIO), Madrid 28029, Spain; ${ }^{5}$ Science for Life Laboratory, Division of Genome Biology, Department of Medical \\ Biochemistry and Biophysics, Karolinska Institute, S-171 21 Stockholm, Sweden
}

\begin{abstract}
MEK inhibition in combination with a glycogen synthase kinase-3 $\beta$ (GSK3 $\beta$ ) inhibitor, referred as the $2 \mathrm{i}$ condition, favors pluripotency in embryonic stem cells (ESCs). However, the mechanisms by which the $2 \mathrm{i}$ condition limits ESC differentiation and whether RAS proteins are involved in this phenomenon remain poorly understood. Here we show that RAS nullyzygosity reduces the growth of mouse ESCs (mESCs) and prohibits their differentiation. Upon RAS deficiency or MEK inhibition, ERF (E twenty-six 2 [Ets2]-repressive factor), a transcriptional repressor from the ETS domain family, translocates to the nucleus, where it binds to the enhancers of pluripotency factors and key RAS targets. Remarkably, deletion of Erf rescues the proliferative defects of RAS-devoid mESCs and restores their capacity to differentiate. Furthermore, we show that Erf loss enables the development of RAS nullyzygous teratomas. In summary, this work reveals an essential role for RAS proteins in pluripotency and identifies ERF as a key mediator of the response to RAS/MEK/ERK inhibition in mESCs.
\end{abstract}

[Keywords: 2i; ERF; pluripotency; RAS; mESCs]

Supplemental material is available for this article.

Received November 27, 2017; revised version accepted March 12, 2018.

RAS proteins are small GTPases that are essential regulators of proliferation, differentiation, and survival in eukaryotic cells (Cox and Der 2010; Pylayeva-Gupta et al. 2011). These proteins oscillate between an active (GTPbound) and inactive (GDP-bound) state that is in turn regulated by guanine exchange factors (GEFs; which promote the GDP/GTP exchange) and GTPase-activating proteins (GAPs; which accelerate GTP hydrolysis). RAS signaling initiates at the membrane, where it integrates cues coming from a wide range of mitogens such as epidermal growth factor (EGF) or fibroblast growth factors (FGF) through their cognate receptors. Upon activation, RAS proteins elicit their function by triggering several phosphorylation-based signaling pathways from which the $\mathrm{RAF} / \mathrm{MEK} / \mathrm{ERK}$ and $\mathrm{PI} 3 \mathrm{~K} / \mathrm{mTOR} / \mathrm{AKT}$ routes are the most studied. Ultimately, the RAS signal is executed by transcription factors, including FOS, JUN, MYC, and factors from the "E twenty-six" (ETS) domain family (Sizemore et al. 2017). While ETS domain factors are best known as activators of transcription, there are also examples of transcriptional repression. One such case is ERF (Ets2-repressive factor), which, in the presence of growth factors, is kept inactive in the cytoplasm by ERK-depen-

Corresponding authors: ofernandez@cnio.es, sruizm@cnio.es Article published online ahead of print. Article and publication date are online at http://www.genesdev.org/cgi/doi/10.1101/gad.310086.117. dent phosphorylation and only translocates to the nucleus upon ERK inactivation to exert its function (Sgouras et al. 1995; Le Gallic et al. 1999).

Besides its well-established roles in regulating proliferation and survival in somatic cells, work with pluripotent cells has also revealed an important role of RAS signaling in promoting differentiation. Mouse embryonic stem cells (mESCs ) are characterized by their indefinite capacity to self-renew and differentiate into all cell types of the organism. In order to maintain their pluripotency, mESCs are cultured in the presence of leukemia inhibitory factor (LIF) and either bone morphogenetic protein 4 (BMP4) or fetal bovine serum (FBS) (Smith et al. 1988; Williams et al. 1988; Ying et al. 2003). Interestingly, ectopic expression of an activated H-RAS in mESCs leads to trophoectodermal differentiation, whereas interfering with FGF4-dependent MEK or ERK signaling impairs neuronal differentiation (Kunath et al. 2007; Lu et al. 2008). These experiments suggested that the RAS/MAPK pathway could be a primary trigger of cell commitment in ESCs. Accordingly, a combined inhibition of MEK and glycogen

(C) 2018 Mayor-Ruiz et al. This article is distributed exclusively by Cold Spring Harbor Laboratory Press for the first six months after the full-issue publication date (see http://genesdev.cshlp.org/site/misc/terms.xhtml). After six months, it is available under a Creative Commons License (Attribution-NonCommercial 4.0 International), as described at http://creativecommons.org/licenses/by-nc/4.0/. 
synthase kinase- $3 \beta$ (GSK3 $\beta$ ), also known as the $2 \mathrm{i}$ condition, is sufficient to maintain pluripotency even in the absence of LIF and serum (Ying et al. 2008). However, regardless of the role of MEK, to what extent RAS proteins are involved in the exit from pluripotency has not been formally addressed. Our work presented here reveals an essential role of RAS proteins in the exit from pluripotency and identifies ERF as a key mediator of the response to RAS/MEK/ERK inhibition in mESCs.

\section{Results}

The absence of RAS genes reduces the growth of MESCs

To investigate the impact of RAS deficiency in pluripotent cells, we generated mESCs carrying constitutively null alleles for $\mathrm{H}$-Ras and N-Ras and a conditional knockout $\mathrm{K}-$ Ras allele $\left(\mathrm{H}-\mathrm{Ras}^{-1-} ; \mathrm{N}-\mathrm{Ras}^{-1-}\right.$; K-Ras ${ }^{\text {lox/lox }}$, referred to here as RAS ${ }^{\text {lox/lox}}$ ) (Drosten et al. 2010). When combined with a Cre ${ }^{\text {ERT2 }}$ expressed from the locus of the large subunit of RNA polymerase II (Brocard et al. 1997), this system allows for the ablation of the remaining $\mathrm{K}$-Ras allele upon addition of 4-hydroxytamoxifen (OHT) and thus the generation of mESCs devoid of all RAS proteins (RAS $\left.{ }^{\text {less }}\right)$. The efficiency of the system was confirmed by Western blotting, which showed an efficient depletion of RAS after exposing RAS ${ }^{\text {lox/lox }}$ mESCs to OHT, together with a severe reduction in the phosphorylation levels of MEK, ERK, and ribosomal S6 kinase (p90RSK), a well-established ERK target (Fig. 1A). In addition, gene set enrichment analysis (GSEA) from microarray data revealed an overall down-regulation of ERK/MAPK targets in OHT-treated RAS $^{\text {lox/lox }}$ mESCs (Fig. 1B; Supplemental Table 1). While MEK inhibition does not severely impair ESC growth (Li et al. 2007), the loss of all RAS proteins significantly reduced the size of mESC colonies (Fig. 1C), suggesting that Ras nullyzygosity could have a more profound impact on MEK/ERK signaling than chemical MEK inhibition. In support of this, the reduction in the phosphorylation levels of ERK and p90RSK was higher in RAS ${ }^{\text {less }}$ cells than in mESCs treated with the MEK inhibitor PD0325901 (Supplemental Fig. S1A). Furthermore, while MEK inhibition
A

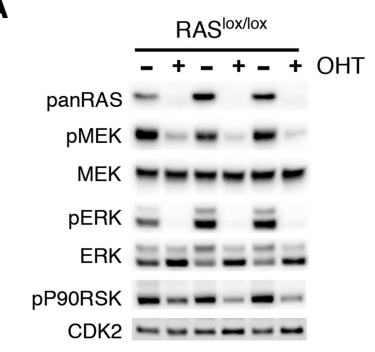

C

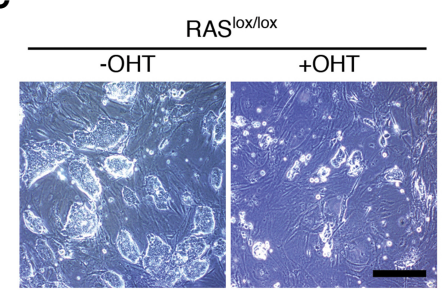

E

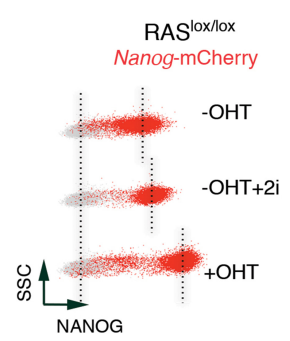

$\mathbf{F}$
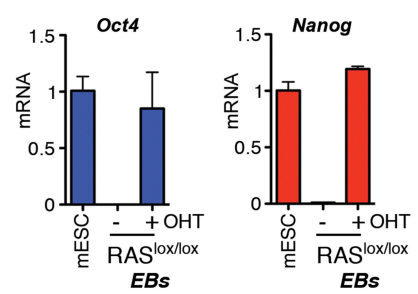

G
B

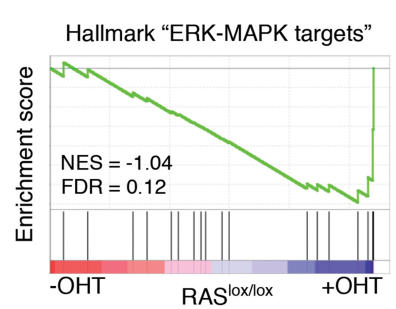

D
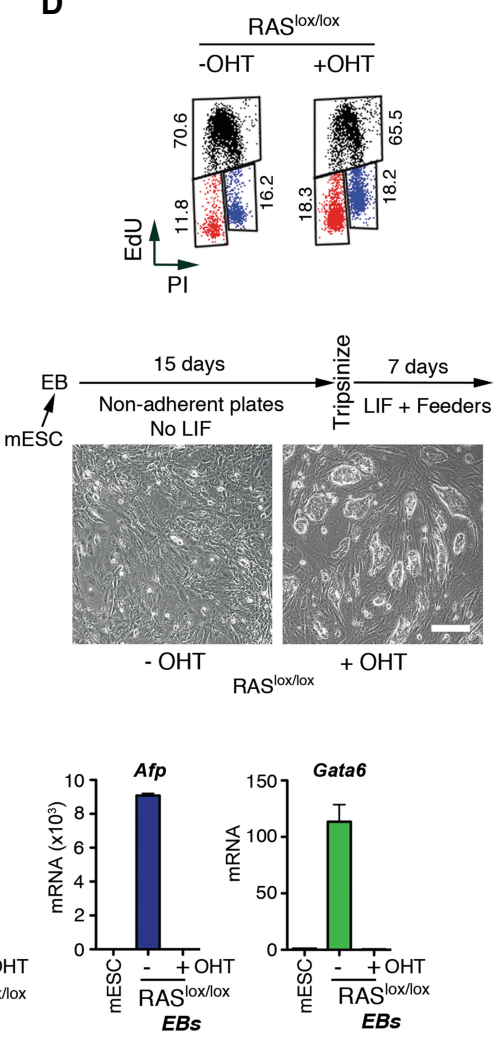

Figure 1. RAS deficiency impairs growth and differentiation in mESCs. (A) Western blot analysis illustrating the loss of the remaining K-RAS protein (with a panRAS antibody) and the decrease in the active phosphorylated forms of MEK, ERK, and P90RSK in OHT-treated (7 d) RAS ${ }^{\text {lox/lox }}$ mESCs. Three independent RAS ${ }^{\text {lox } / l o x}$ mESC lines were used. CDK2 levels are shown as a loading control. (B) Preranked GSEA on the genes included in the hallmark "ERK-MAPK targets" revealed a down-regulation of the pathway in RAS-deficient mESCs. The heat map representation derives from two independent untreated or OHT-treated RAS ${ }^{\text {lox } / \mathrm{lox}} \mathrm{mESC}$ lines $2 \mathrm{~d}$ after the addition of OHT. (C) Representative bright-field images corresponding to untreated or OHT-treated RAS ${ }^{\text {lox/lox }}$ mESCs $7 \mathrm{~d}$ after the addition of OHT. Bar, $20 \mu \mathrm{m}$. (D) Flow cytometry analysis of the cell cycle distribution in untreated or OHT-treated RAS ${ }^{\text {lox/lox }}$ mESCs $7 \mathrm{~d}$ after the addition of OHT. (E) Flow cytometry analysis of a knocked-in mCherry reporter at the Nanog locus in RAS ${ }^{\text {lox } / \text { lox }}$ mESCs untreated, treated with OHT, and treated with $2 \mathrm{i}$ for 3 d. $(F)$ mRNA levels of pluripotent (Oct4 and Nanog) and differentiation (Afp and Gata6) markers in wild-type mESCs and in embryoid bodies (EBs) derived from untreated or OHT-treated RAS ${ }^{\text {lox/lox }}$ mESCs as assessed by real-time PCR. Gapdh levels were used to normalize gene expression. Two independent experiments were performed, and data are shown as the averaged level of triplicates. Error bars indicate SD. $(G)$ Representative brightfield images of the cultures initiated from EBs derived from untreated or OHT-treated RAS ${ }^{\text {lox/lox }}$ mESCs that were maintained in suspension and in the absence of LIF for 15-d and subsequently trypsinized and plated on top of feeder mouse embryonic fibroblasts (MEFs) in mESC medium. Bar, $20 \mu \mathrm{m}$. 
triggers a feedback loop that results in increased MEK phosphorylation (Caunt et al. 2015), the phosphorylation levels of MEK are sharply reduced in RAS ${ }^{\text {less }}$ cells (Supplemental Fig. S1A).

To analyze the growth properties of RAS ${ }^{\text {less }}$ mESCs, OHT-treated and untreated RAS ${ }^{\text {lox/lox }}$ cells were infected with H2B-EGFP-expressing lentiviruses and video-monitored for $24 \mathrm{~h}$ at $10-\mathrm{min}$ intervals. This analysis revealed that the reduced proliferation of RAS ${ }^{\text {less }}$ cells was not due to an increase in cell death but rather the presence of cells that failed to progress into mitosis, consistent with the key role of MAPK signaling as a mitogenic pathway (Supplemental Fig. S1B). Flow cytometry analyses also did not show an increase of cell death in RAS less mESCs but revealed a slight arrest at the G1/S boundary (Fig. 1D). This phenotype is reminiscent of recent observations made in mESCs grown in $2 \mathrm{i}$ (Ter Huurne et al. 2017).

\section{RAS deficiency prohibits mESC differentiation}

Next, we investigated the effects of RAS deficiency in pluripotency. Similar to the "ground state" achieved with the $2 \mathrm{i}$ condition (Ying et al. 2008), RAS ${ }^{\text {less }}$ mESCs presented a more intense and homogeneous expression of NANOG and fewer differentiated colonies based on alkaline phosphatase activity (Supplemental Fig. S1C,D). Accordingly, flow cytometry analysis of a knocked-in mCherry reporter at the Nanog locus (Faddah et al. 2013) revealed that the loss of RAS genes in mESCs led to an increase in NANOG expression, which was actually higher than that achieved with the $2 \mathrm{i}$ condition (Fig. 1E). To evaluate the capacity of RAS-deficient mESCs to undergo differentiation, we first induced their differentiation toward intermediate mesoderm with a protocol based on retinoic acid and activin A (Oeda et al. 2013). This experiment revealed that RAS $^{\text {less }}$ mESCs induced to differentiate retained the expression of the pluripotency factors Oct4 and Nanog and failed to express differentiation markers such as $A f p$, Foxa2, and Msx1 (Supplemental Fig. S1E).

To further induce differentiation, we generated embryoid bodies (EBs) from OHT-treated or untreated RAS ${ }^{\text {lox/lox }}$ mESCs and subsequently transferred them to gelatincoated plates where they were left to differentiate for 10 additional days. Remarkably, even if RAS ${ }^{\text {less }}$ mESCs were capable of generating EBs (see examples below), these EBs maintained the expression of Oct4 and Nanog and failed to express differentiation markers such as Gata4, Afp, Foxa2, Gata6, and Albumin (Fig. 1F; data not shown). Finally, we tried to promote the differentiation of RAS ${ }^{\text {less }}$ mESCs with the following experiment. EBs derived from untreated and OHT-treated RAS ${ }^{\text {lox } / \text { lox }}$ mESCs were transferred to nonadherent plates in the absence of LIF and kept in suspension for $15 \mathrm{~d}$, which is a highly stringent protocol, after which no pluripotent cells should remain. After this time, EBs were tripsinized and transferred to regular mESC culture plates containing feeders and LIF. As expected, no mESC colonies grew from trypsinized RAS-proficient EBs. In contrast, cultures initiated from RAS ${ }^{\text {less }}$ EBs yielded abundant mESC colo- nies, confirming the incapacity of RAS-deficient mESCs to differentiate (Fig. 1G). Importantly, the lack of H-Ras and N-Ras present in RAS ${ }^{\text {lox/lox }}$ cells does not significantly affect the self-renewal or differentiation capabilities of mESCs (Supplemental Fig. S2). Altogether, these experiments illustrate that the absence of RAS genes reduces the growth of mESCs and prevents their exit from pluripotency.

\section{ERF deletion rescues growth and differentiation in $R A S^{\text {less }} \mathrm{mESCS}$}

In mammals, cumulative evidence supports an important role for ERF in counteracting the RAS signal (Sgouras et al. 1995; Le Gallic et al. 1999; Verykokakis et al. 2007). ERF is a transcriptional repressor from the ETS family that, in the presence of an active RAS/MEK/ERK pathway, is kept inactive in the cytoplasm through ERK-dependent phosphorylation and translocates to the nucleus upon ERK inhibition, leading to cell cycle arrest at G0/G1 (Le Gallic et al. 1999, 2004). Interestingly, ERF expression is particularly high in mESCs when compared with other cell types (Supplemental Fig. S3), suggesting a distinct function for this ETS domain factor in pluripotency. In support of this, exposure of mESCs to the $2 \mathrm{i}$ condition or the loss of Ras genes promoted the nuclear accumulation of ERF (Fig. 2A; Supplemental Fig. S4A,B). In addition, ERF phosphorylation levels were reduced in RAS ${ }^{\text {lox/lox }}$ mESCs upon OHT treatment, further indicating its activation in RAS less cells (Fig. 2B). To evaluate whether ERF played a role in the reduced growth rates of RAS less cells, RAS ${ }^{\text {lox/lox }}$ mESCs were infected with lentiviruses expressing Cas9 and two different single-guide RNAs (sgRNAs) targeting Erf. CRISPR-mediated depletion of ERF was efficient with both sgRNAs, as determined by Western blotting (Fig. 2B). ERF depletion fully rescued the growth of RAS ${ }^{\text {less }}$ mESCs (Fig. 2C; Supplemental Fig. $\mathrm{S} 4 \mathrm{C})$. Video analysis of cell cycle progression confirmed the rescue of proliferation and mitotic entry rates that is achieved by Erf deletion in RAS-deficient mESCs (Supplemental Fig. S4D).

Next, we investigated whether ERF depletion could enable the differentiation of mESCs devoid of RAS genes by generating EBs. Consistent with the growth defects observed in RAS ${ }^{\text {less }}$ cells, EBs generated from OHT-treated $\mathrm{RAS}^{\mathrm{lox} / \mathrm{lox}} \mathrm{mESC}$ were smaller in size than RAS-proficient ones, an effect that was once again rescued by ERF depletion (Fig. 2D; Supplemental Fig. S4E). Subsequently, EBs were cultured on gelatin-coated plates, which leads to the growth of patches of differentiated cells that spread as a layer away from the EBs (Fig. 2E). In agreement with their incapacity to differentiate, RAS ${ }^{\text {less }}$ EBs did not present such an outgrowth and stayed as compact EBs. In contrast, layers of emigrating cells were observed in the periphery of RAS ${ }^{\text {less }}$ EBs that were also deficient in ERF (Fig. 2E), which expressed differentiation markers such as NESTIN (Supplemental Fig. S4F). ERF loss enabled a high degree of differentiation in RAS ${ }^{\text {less }}$ cells, as exemplified by the observation of beating cardiomyocytes emerging from ERFdeficient RAS ${ }^{\text {less }}$ EBs (Supplemental Movie 1). 

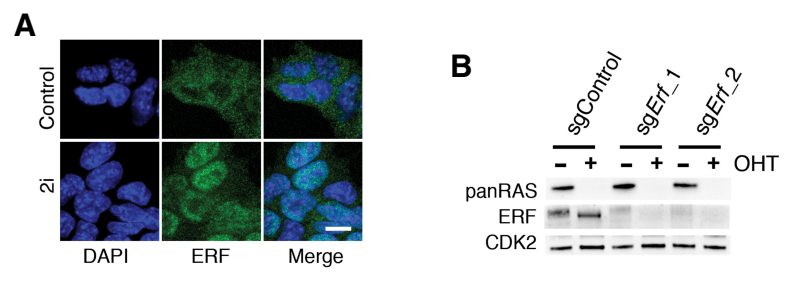

C
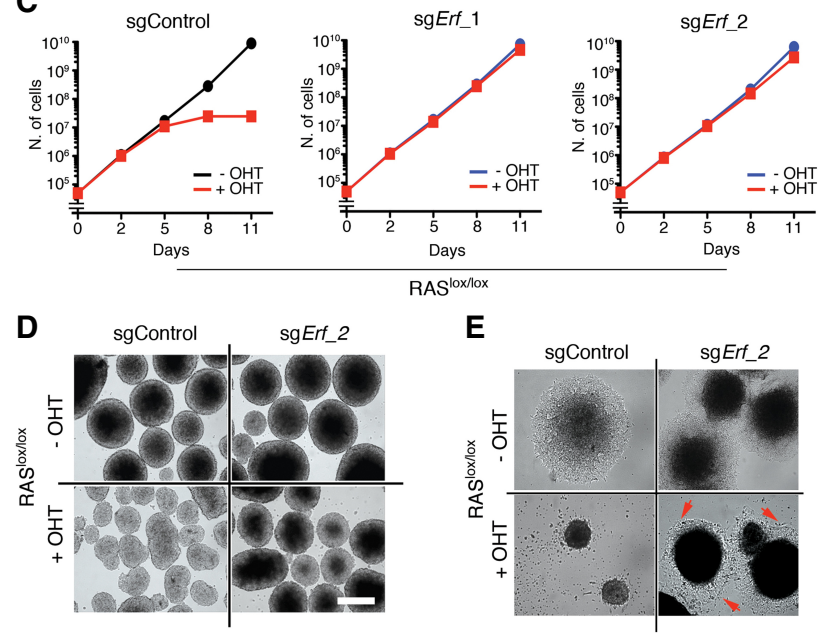

Figure 2. ERF loss rescues the effecs of RAS deficiency in mESCs. (A) Intracellular localization of ERF (green) in control or 2i-treated ( $2 \mathrm{~h}$ ) wild-type mESCs. Bar, $5 \mu \mathrm{m}$. DAPI (blue) was used to stain DNA. (B) Western blot of ERF observed in untreated or OHT-treated $(48 \mathrm{~h}) \mathrm{RAS}^{\text {lox } / \mathrm{lox}}$ mESCs that had been infected with lentiviruses expressing Cas9 and sgRNAs against ERF (sgErf_1 and sgErf_2) or a control sgRNA. The ERF band shift that occurs upon loss of all Ras proteins (detected with a panRAS antibody) is consistent with a loss of phosphorylation. CDK2 levels are shown as a loading control. (C) Cumulative growth curves from cultures of untreated or OHT-treated RAS ${ }^{\text {lox/lox }}$ mESCs infected with lentiviruses expressing Cas 9 and sgRNAs against ERF (sgErf_1 and sgErf_2) or a control sgRNA. (D) Representative bright-field images from 4-d-old EBs maintained in suspension derived from untreated or OHT-treated RAS ${ }^{\text {lox/lox }}$ mESCs infected with lentiviruses expressing Cas9 and sgRNAs against ERF (\#2) or a control sgRNA. EB formation was initiated $5 \mathrm{~d}$ after the addition of OHT. Bar, $100 \mu \mathrm{m}$. (E) Representative bright-field images of 6-d-old EBs derived from untreated or OHT-treated RAS ${ }^{\text {lox/lox }}$ mESCs infected with lentiviruses expressing Cas9 and sgRNAs against $\operatorname{Erf}(\# 2)$ or a control sgRNA that were subsequently plated onto gelatin to induce spontaneous differentiation. Bar, $100 \mu \mathrm{m}$.

\section{ERF deletion enables the development of RAS nullyzygous teratomas}

Besides their role in pluripotency, RAS genes are best known as the most frequently mutated human oncogene (Pylayeva-Gupta et al. 2011). Given that RAS structural properties are unfavorable for the development of small molecule inhibitors (Cox et al. 2014), current therapies are oriented to target downstream effectors of RAS, such as RAF or MEK. However, resistance to these treatments invariably occurs due to the existence of numerous feedback loops (Samatar and Poulikakos 2014). In this context, even if potent and selective RAS inhibitors finally emerge, to what extent the anti-tumoral effects of these agents could also be bypassed by resistance mechanisms is not known. To evaluate the tumor formation potential of RAS-deficient mESCs, we generated teratomas. To this end, untreated and OHT-treated RAS ${ }^{\text {lox/lox }}$ mESCs were injected subcutaneously into both flanks of nude mice. To discard variability between host mice, untreated mESCs were injected into one flank, and OHT-treated ones were injected into the other. Consistent with the major role of RAS in cancer, OHT-treated RAS ${ }^{\text {lox } / \text { lox }}$ mESCs failed to form teratomas (Fig. 3A-C). In contrast, Erf deletion enabled the development of RAS-deficient teratomas (Fig. 3A-C). The size of ERF-deficient RAS ${ }^{\text {less }}$ teratomas was similar to that of control tumors, further illustrating the extent of the synthetic viable effect that arises upon the concomitant loss of ERF and RAS (Fig. 3B; Supplemental Fig. S5A). Interestingly, one teratoma could be obtained from RAS ${ }^{\text {less }}$ mESCs that were ERF wild type, which turned out to be a chimaera of RAS-proficient and RAS-deficient cells due to an incomplete OHT-induced deletion of the remaining K-Ras allele (Fig. 3D). Consistent with the incapacity of RAS-deficient mESCs to
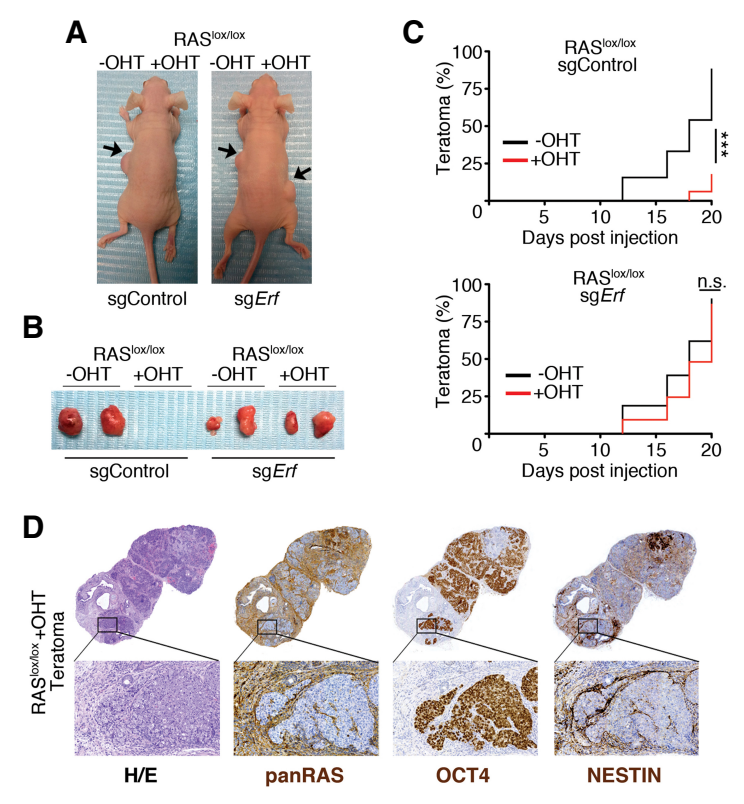

Figure 3. ERF deletion enables the development of RAS-devoid teratomas. $(A)$ Representative images of mice bearing teratomas $20 \mathrm{~d}$ after the injection of untreated or OHT-treated RAS ${ }^{\text {lox/lox }}$ mESCs infected with lentiviruses expressing Cas9 and sgRNAs against ERF or a control sgRNA. Eight mice were injected per condition. (B) Representative images of the teratomas obtained from the experiment defined in $A$ at day 20. $(C)$ Kaplan-Meier graphs illustrating the percentage of mice bearing teratomas after the injection of the indicated mESCs. (n.s.) Nonsignificant; $\left(^{* * *}\right) P<$ 0.001. (D) Immunohistochemistry of panRAS, OCT4, and NESTIN expression in the only teratoma obtained from OHT-treated RAS ${ }^{\text {lox/lox }}$ mESCs that had been infected with a control sgRNA. Note that areas lacking panRAS staining retained OCT4 expression and failed to express NESTIN (zoomed-in square). 
undergo differentiation, areas of this teratoma lacking RAS expression retained high levels of OCT4 and failed to express NESTIN, while differentiation could be readily observed in RAS-positive areas. Upon ERF loss, NESTINpositive and OCT4-negative areas could be observed in RAS-deficient teratomas (Supplemental Fig. S5B). Moreover, ERF-deficient RAS nullyzygous teratomas presented areas of differentiation into all three embryonic layers (Supplemental Fig. S5C). In summary, these data demonstrate that Erf deletion enables the differentiation of mESCs devoid of all Ras genes in vitro and in vivo.

\section{ERF is a regulator of transcriptional enhancers in mESCs}

To understand the mechanism by which ERF regulates growth and differentiation in mESCs, we mapped its genomic binding sites by chromatin immunoprecipitation (ChIP) followed by next-generation sequencing (ChIPseq). To this end, we analyzed the chromatin association of ERF in RAS ${ }^{\text {lox/lox }}$ mESCs that had been either treated or untreated with OHT for $7 \mathrm{~d}$ as well as in ERF-deficient mESCs as an antibody specificity control (Supplemental Fig. S6A). Consistent with the cytoplasmic localization of ERF in RAS-proficient cells, only one ERF peak could be detected in this condition (Supplemental Table 2). In contrast, 4297 potential ERF-binding sites were detected in OHT-treated RAS ${ }^{\text {lox/lox }}$ mESCs (Supplemental Table 3). Interestingly, while ETS domain factors are most often associated with transcriptional control at promoter sites, a detailed analysis of ERF-bound sequences in Ras less mESCs showed that $45.5 \%$ of the peaks map to sequences 10-100 kb away from the transcription start site (TSS) (Fig. 4A). Moreover, the majority of ERF peaks is at either introns $(36 \%)$ or intergenic sequences (45\%) (Fig. 4B), similar to previous observations from an ERF ChIP-seq in growth-deprived mouse embryonic fibroblasts (MEFs) (Twigg et al. 2013).

The data above prompted us to explore whether ERF binding could preferentially occur at transcriptional enhancer sequences. To this end, we compared its distribution with a reference data set of 10,627 mESC enhancers (Hnisz et al. 2013), identifying ERF peaks in 1954 of these sites (Fig. 4C). Consistently, an independent statistical analysis identified that $49.1 \%$ all ERF peaks have a $P$-value of $<0.05$ for overlapping with $\mathrm{mESC}$ enhancer sequences (vs. randomly distributed) (see the Supplemental Material). Moreover, the distribution of ERF at these sites was similar to that of mESC enhancer-associated marks such as H3K4me1 and P300 (Fig. 4D,E; Heintzman et al. 2007). Interestingly and in addition to "negative regulation of MAPK cascade," gene ontology analyses identified "stem cell maintenance" and "blastocyst formation" among the most significantly enriched pathways harboring ERF-bound enhancers, highlighting a key role for ERF in the coordination of pluripotency (Supplemental Fig. S6B; Supplemental Table 4). In support of this, ERF bound to enhancer sequences present near the genes from core pluripotency factors such as OCT4 and KLF4 as well as from other factors with an important role in the maintenance of the ground state, such as ESSRB,
A
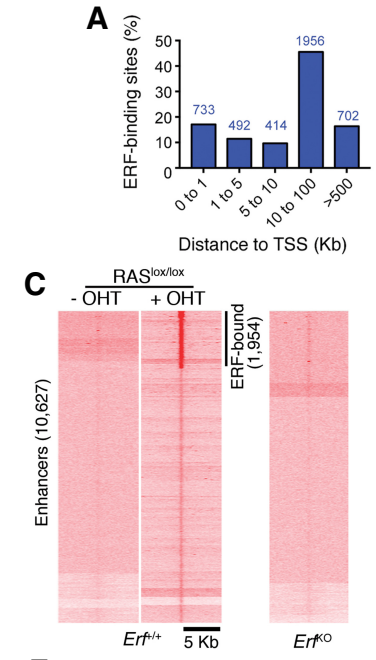

F
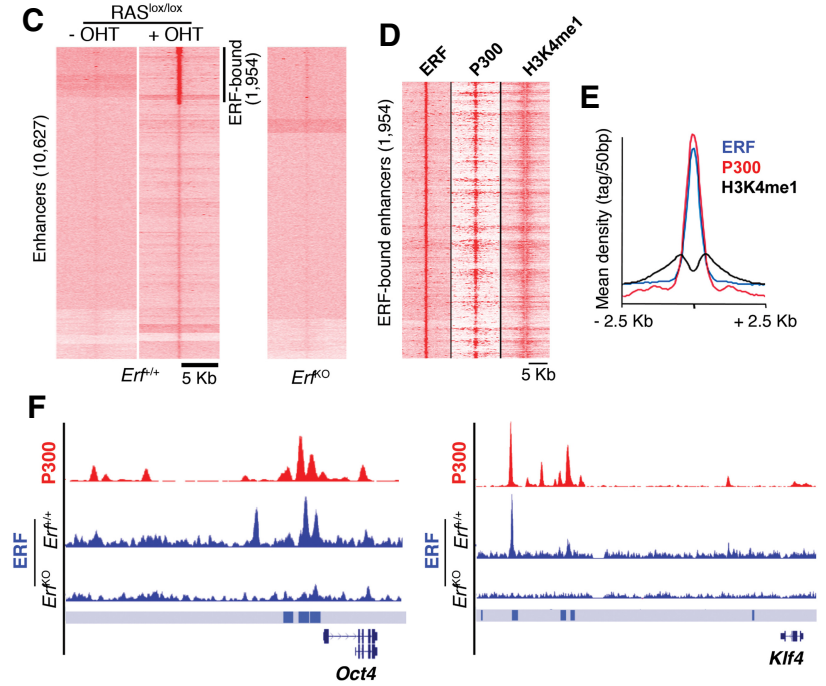

Figure 4. ERF binds to enhancers of RAS targets and pluripotency factors in mESCs. (A) Distribution of ERF-bound genomic regions in RAS-deficient mESCs related to the closest TSS. Numbers within the graph correspond to the number of ERF-bound genomic regions included in each category. $(B)$ Distribution of the different genomic features overlapping with the 4297 ERF peaks identified in RAS ${ }^{\text {less }}$ mESCs, identified with the HOMER bioinformatics package (see the Supplemental Material). (C) Heat map representations from untreated and OHT-treated RAS ${ }^{\text {lox/lox }}$ mESCs of the normalized read density of the ERF ChIP-seq at 10,627 previously defined mESC enhancers (Hnisz et al. 2013). The heat map from the ERF ChIP-seq in Erf ${ }^{\mathrm{KO}}$ cells is provided as a control. (D) Heat map representation of the normalized read density of ERF ChIP-seq data around the 1954 enhancers bound by ERF in OHT-treated RAS ${ }^{\text {lox/lox }}$ mESCs. The overlap with public ChIP-seq data sets of P300 (Gene Expression Omnibus [GEO] accession no. GSM918750) and H3K4me1 (GEO accession no. GSM1003750) distributions in mESCs is shown. (E) Average ERF, P300, and H3K4me1 ChIP-seq mean density distributions in mESCs as defined in $D$, centered around enhancers. $(F)$ Representative ChIP-seq tracks of ERF at the Oct4 (left) and Klf4 (right) locus in RAS ${ }^{\text {less }}$ mESCs. The ChIP-seq signal from ERF-deficient $\mathrm{mESC}$ is also provided as a specificity control. The $X$-axis corresponds to the genomic location, and the $Y$-axis corresponds to normalized ChIP-seq signal density. The ChIP-seq track of $\mathrm{P} 300$ in mESCs (GEO accession no. GSM918750) is provided for comparison with a reference enhancer-associated mark. Known mESC enhancers are shown as blue boxes in the bottom track.

NR5A2, PRDM14, TCFCP2L1, STAT3, and TCF3 (Fig. 4F; Hnisz et al. 2013; data not shown). Moreover, while motif analyses revealed ETS-binding sites as the most significantly enriched sequences at ERF peaks, this was followed in significance by the target sequences for OCT4, SOX2, and KLF4 (Supplemental Fig. S6C). 
Regarding the impact of ERF binding in gene expression, bioinformatics analysis with the genomic enrichment of annotations tool (GREAT) (McLean et al. 2010) identified 5098 genes as associated with ERF peaks. When comparing this data set with microarray analyses of OHT-treated and untreated RAS ${ }^{\text {lox/lox }}$ mESCs (Supplemental Table 1), 468 out of the 5098 genes $(9.2 \%)$ that lay near ERF peaks were found to be down-regulated in RAS-deficient mESCs (Supplemental Table 5), consistent with the known role of ERF as a transcriptional repressor. Accordingly, ERF deletion increased the expression of the majority of genes that are down-regulated in RAS less mESCs and are in close proximity to ERF-bound enhancer regions (Supplemental Fig. S6D). This is exemplified by JARID2 or MYC, for instance, which present ERF peaks in the vicinity of their genes and are down-regulated in the absence of RAS in a manner that is alleviated by ERF deletion (Supplemental Fig. S6E-H). Besides Myc, the list of genes that present ERF peaks and are down-regulated in RAS-deficient mESCs includes key transducers of the RAS signal, such as $P d k 1$, the ETS domain factors Etv1 and Etv4, and several negative regulators of growth factor signaling, including Spred1, Dusp4, Dusp6, Spry2, and Spry4. (Supplemental Table 5). Interestingly, 284 out of the 5098 genes $(5.6 \%)$ associated with ERF peaks in RAS ${ }^{\text {less }}$ mESCs presented increased expression in these cells, suggesting that ERF might also play an activating role at certain loci (Supplemental Table 6). In support of this, ERF deletion reduced the expression levels of many of the genes that are up-regulated in RAS ${ }^{\text {less }}$ mESCs and are in close proximity to ERF-bound enhancers (Supplemental Fig. S6I). Thus, despite the well-established role of ERF as a transcriptional repressor (Sgouras et al. 1995; Le Gallic et al. 1999; Verykokakis et al. 2007), our current data indicate that the presence of ERF at mESC enhancers can either activate or repress transcription of the associated gene. Collectively, these data identify ERF as an enhancer-bound regulator of gene expression in pluripotent cells.

\section{Discussion}

While RAS proteins are mostly known as oncogenic factors, the RAS/MEK/ERK axis also plays a key role in pluripotency. Accordingly, MEK inhibition or ERK1/2 deficiency limits the differentiation of mESCs (Ying et al. 2008; Chen et al. 2015). Our work further supports this concept, as mESCs devoid of RAS genes are completely incapable of abandoning pluripotency in vitro or in vivo. Still, how the inhibition of growth factor signaling favors pluripotency remains poorly understood. Here we identify ERF as member of the ETS family of transcription factors with a key role in this phenomenon. Upon loss of RAS proteins, ERF translocates to the nucleus, where it binds to a broad set of enhancers placed near key pluripotency and mitogenic factors. The localization of ERF at enhancers is consistent with recent ChIP-seq studies on ETS factors (Chen et al. 2013; Yang et al. 2015), which could indicate that this metazoan-specific family of transcriptional regulators might have evolved together with the emergence of enhancer sequences as modulators of their function. Given that ETS factors share a common binding sequence, it is possible that the presence of ERF upon inhibition of the RAS/MEK/ ERK pathway impedes the recruitment of other ETS factors, thereby limiting their function.

The central role that ERF plays in restricting proliferation and differentiation in mESCs is unlikely to be determined by a single factor. Similar to what was reported for the $2 \mathrm{i}$ condition (Marks et al. 2012), reduced levels of MYC probably play an important role in the growth defects of RAS ${ }^{\text {less }}$ mESCs. Accordingly, previous work identified MYC as a mediator of the growth-suppressing functions of ERF in MEFs (Verykokakis et al. 2007). Nevertheless, ERF binds to the enhancers of multiple members of the growth factor signaling pathway and ETS domain factors, some of which probably also contribute to the reduced growth rates of RAS ${ }^{\text {less }}$ mESCs. As to how ERF restricts mESC differentiation, the polycomb repression complex 2 (PRC2) subunit JARID2 is an interesting candidate. Multilineage differentiation in mESCs depends on the transcriptional priming of developmental bivalent (H3K4me3/H3K27me3) genes by JARID2. Accordingly, JARID2 knockout mESCs have a severe compromised capacity to differentiate and are unable to intiate cell lineage commitment (Landeira et al. 2010). In this context, the finding that JARID2 levels are significantly down-regulated in RAS-deficient mESCs in an ERF-dependent manner provides an interesting mechanistic insight that could help in understanding how inhibition of the RAS/MEK/ ERK pathway promotes a more ground state in mESCs. Besides the role of individual targets, ERF might also play a more general role in limiting mESC differentiation. Recent works have revealed that the transition from naïve to primed pluripotency involves a global reorganization of enhancer usage patterns for factors such as OCT4, which relocates from distal enhancers in naïve mESCs to more proximal enhancers in primed cells (Buecker et al. 2014; Factor et al. 2014). Likewise, OCT4, SOX2, and NANOG translocate to distal enhancers when mESCs are cultured under the $2 \mathrm{i}$ conditions (Galonska et al. 2015), suggesting that the "distal enhancer"-binding profile is a unique feature of ground-state mESCs. Given that ERF-bound enhancers are mostly distal, to what extent ERF binding mediates the global rewiring of enhancer usage associated with pluripotency emerges as an interesting possibility.

In regard to cancer, our work opens the unfortunate possibility that even if potent and selective RAS targeting drugs are finally developed, resistance mechanisms are likely to emerge. Supporting this concept, a recent study has shown that some pancreatic cancer lines can tolerate the absence of K-RAS (Muzumdar et al. 2017), although there could potentially be compensation from the rest of the members of the RAS family (Esteban et al. 2001; Potenza et al. 2005; Drosten et al. 2017). It is noteworthy that while $\mathrm{H}-R A S, \mathrm{~N}-R A S$, and $\mathrm{K}-R A S$ are widely considered the main $R A S$ genes in mammalian cells, mESCs express another variant, E-Ras, that could drive RAS signaling in pluripotent cells (Takahashi et al. 2003). However, the profound inhibition of MEK and ERK 
phosphorylation in RAS ${ }^{\text {less }}$ mESCs argues against E-Ras significantly contributing to RAS signaling in these cells (Fig. 1A; Supplemental Fig. S1A). In this context, while acknowledging that teratomas are a special tumor type, our data suggest that cancer cells might even be able to bypass RAS nullyzygosity through the inactivation of suppressors such as ERF. Interestingly, recurrent deletions and inactivating mutations of $E R F$ have been found recently in prostate cancer (Bose et al. 2017; Dhingra et al. 2017; Huang et al. 2017). However, the impact of these mutations is still not fully characterized. Based on our work, it is tempting to speculate that loss of ERF function might increase the resistance to targeted therapies of the RAS/ MEK/ERK pathway, including actual RAS inhibitors. Beyond the putative impact of ERF mutations in cancer, our work places ERF at the core of the response to RAS/ MEK/ERK inhibition in pluripotent cells.

\section{Materials and methods}

Mice

Athymic nude Foxn1-null mice were obtained from Charles River. All mouse work was performed in the pathogen-free animal facility of the Spanish National Cancer Research Centre (CNIO) in accordance with the Guidelines for Humane Endpoints for Animals Used in Biomedical Research and under the supervision of the Ethics Committee for Animal Research of the "Instituto de Salud Carlos III."

Cell lines

$\mathrm{N}-\mathrm{Ras}^{-1-}$; H-Ras ${ }^{-/-}$; K-Ras ${ }^{f / f}$; Ubiq-Cre ${ }^{E R T 2}$ (Drosten et al. 2010) and wild-type (R1 and G4) mESCs were grown on a feeder layer of growth-arrested MEFs or on gelatin at $37^{\circ} \mathrm{C}$ and $5 \% \mathrm{CO}_{2}$ in highglucose DMEM (Invitrogen) supplemented with 15\% FBS, 1000 $\mathrm{U} / \mathrm{mL}$ LIF, $0.1 \mathrm{mM}$ nonessential amino acids, $1 \%$ glutamax, 55 $\mathrm{mM} \beta$-mercaptoethanol, and $1 \%$ penicillin/streptomycin unless otherwise indicated. MEFs were obtained from 13.5 embryos by standard methods. HEK293T (American Type Culture Collection) cells were grown in DMEM, 10\% FBS, and 1\% penicillin/ streptomycin.

\section{mESC differentiation}

For differentiation toward intermediate mesoderm, dissociated $\mathrm{RAS}^{\text {lox/lox }}$ mESCs (untreated or OHT-treated previously for at least $5 \mathrm{~d}$ ) were seeded without feeders on gelatin-coated plates and cultured in DMEM supplemented with $10 \%$ FBS, $0.1 \mathrm{mM}$ nonessential amino acids, $55 \mathrm{mM} \beta$-mercaptoethanol, $1 \%$ penicillin/streptomycin, and $10 \mathrm{ng} / \mathrm{mL}$ activin $\mathrm{A}$ for $4 \mathrm{~d}$ as described previously (Oeda et al. 2013). In the last $2 \mathrm{~d}, 50 \mathrm{nM}$ retinoic acid was also added to the culture medium.

EBs

To generate EBs, trypsinized ERF-proficient and ERF-deficient RAS ${ }^{\text {lox } / \text { lox }}$ mESCs (untreated or OHT-treated previously for at least 5 d) were hanging drop-cultured at a density of 60,000 cells per milliliter for $2 \mathrm{~d}$. After this time, cells were collected and cultured in suspension in low-attachment plates in DMEM without LIF supplemented with $10 \%$ FBS, $0.1 \mathrm{mM}$ nonessential amino acids, $55 \mathrm{mM} \beta$-mercaptoethanol, and $1 \%$ penicillin/streptomycin. To further induce differentiation, EBs were transferred to gelatin- coated plates and left to differentiate for 10 additional days in the same medium condition.

Teratoma formation

mESCs $\left(1 \times 10^{6}\right)$ were subcutaneously injected into the flanks of nude Foxn1-null mice and inspected daily for a period of several weeks to assess teratoma growth and size (measures were calculated according to the formula length $\times$ width $\left.^{2} \times 0.5\right)$. Eight independent injections per group were performed.

Plasmids

The lentiviral plasmid pLentiCRISPRv2 (Addgene, 52961) was used to express sgRNAs. The sequences of the sgRNAs used were designed with the Massachusetts Institute of Technology CRISPR design tool (http://www.genome-engineering. org/crispr). pLenti-H2B-EGFP was a kind gift from Dr. Marcos Malumbres (CNIO). The plasmid Nanog-2A-mCherry (Addgene, 59995) was used to knock in a mCherry cDNA at the Nanog locus.

\section{High-throughput microscopy}

High-throughput microscopy for the analysis of ERF nuclear translocation was performed as described previously with antibodies against endogenous ERF (see Supplemental Table 7 for a list of antibodies used in this study; Lopez-Contreras et al. 2012). Briefly, images from each well were automatically acquired by an Opera high-content screening system (Perkin Elmer) at nonsaturating settings and segmented using DAPI staining to generate masks matching cell nuclei. Upon quantification of ERF signals, a nuclear/cytoplasmic ratio was established for each cell. Where the observed ratio was above the values found in $95 \%$ of untreated mESCs, the cells were considered to have a "nuclear" localization of ERF. Data were represented with Prism (GraphPad Software).

\section{ChIP-seq and microarray data}

Microarray and ChIP-seq data are available at the NCBI Gene Expression Omnibus under accession number GSE99477. Bioinformatics analyses and experimental methods for ChIP-seq and microarray experiments are detailed in the Supplemental Material.

\section{Acknowledgments}

We thank Cian Lynch, Jorge Monsech, and Diego Megias for their help with microarray, ChIP-seq, and high-throughput microscopy analyses. We also thank Dr. Manuel Serrano and Dr. André Nussenzweig for their input on the manuscript, and Dr. Diego Sanz for his support throughout the project. C.M.-R. was funded by a PhD fellowship from La Caixa Foundation, T.O. was funded by a $\mathrm{PhD}$ fellowship from the Boehringer Ingelheim Fonds, and S.R. was funded by a Ramon y Cajal contract (RYC-2011-09242). Research was funded by Fundación Botín and Banco Santander through its Santander Universities Global Division; grants from the Spanish Ministry of Economy and Competitiveness (SAF2011-23753 and SAF201457791-REDC; these projects were cofinanced with European Fonds Européen de Développement Économique et Régional [FEDER] funds), Fundació La Marato de TV3, Howard Hughes Medical Institute, and the European Research Council (ERC617840) to O.F.-C.; and grants from the Spanish Ministry 
of Economy and Competitiveness (SAF2013-49147-P and SAF2016-80874-P; these projects were cofinanced with European FEDER funds) to S.R.

Author contributions: C.M.-R. and S.R. conducted most of the experiments; T.O. helped with the characterization of RAS ${ }^{\text {lox/lox }}$ mESCs and with ERF localization studies; E.L. helped with ChIP-seq experiments; M.D., S.O., and M.B. contributed to the work on RAS-deficient cells; M.V.-S. provided technical help; O.D. helped with genomics experiments and bioinformatics analysis; and S.R. and O.F.-C. coordinated the study and wrote the manuscript.

\section{References}

Bose R, Karthaus WR, Armenia J, Abida W, Iaquinta PJ, Zhang Z, Wongvipat J, Wasmuth EV, Shah N, Sullivan PS, et al. 2017. ERF mutations reveal a balance of ETS factors controlling prostate oncogenesis. Nature 546: 671-675.

Brocard J, Warot X, Wendling O, Messaddeq N, Vonesch JL, Chambon P, Metzger D. 1997. Spatio-temporally controlled site-specific somatic mutagenesis in the mouse. Proc Natl Acad Sci 94: 14559-14563.

Buecker C, Srinivasan R, Wu Z, Calo E, Acampora D, Faial T, Simeone A, Tan M, Swigut T, Wysocka J. 2014. Reorganization of enhancer patterns in transition from naive to primed pluripotency. Cell Stem Cell 14: 838-853.

Caunt CJ, Sale MJ, Smith PD, Cook SJ. 2015. MEK1 and MEK2 inhibitors and cancer therapy: the long and winding road. Nat Rev Cancer 15: 577-592.

Chen Y, Chi P, Rockowitz S, Iaquinta PI, Shamu T, Shukla S, Gao D, Sirota I, Carver BS, Wongvipat J, et al. 2013. ETS factors reprogram the androgen receptor cistrome and prime prostate tumorigenesis in response to PTEN loss. Nat Med 19: 1023-1029.

Chen H, Guo R, Zhang Q, Guo H, Yang M, Wu Z, Gao S, Liu L, Chen L. 2015. Erk signaling is indispensable for genomic stability and self-renewal of mouse embryonic stem cells. Proc Natl Acad Sci 112: E5936-E5943.

Cox AD, Der CJ. 2010. Ras history: the saga continues. Small GTPases 1: 2-27.

Cox AD, Fesik SW, Kimmelman AC, Luo J, Der CJ. 2014. Drugging the undruggable RAS: mission possible? Nat Rev Drug Discov 13: 828-851.

Dhingra P, Martinez-Fundichely A, Berger A, Huang FW, Forbes AN, Liu EM, Liu D, Sboner A, Tamayo P, Rickman DS, et al. 2017. Identification of novel prostate cancer drivers using RegNetDriver: a framework for integration of genetic and epigenetic alterations with tissue-specific regulatory network. Genome Biol 18: 141.

Drosten M, Dhawahir A, Sum EY, Urosevic J, Lechuga CG, Esteban LM, Castellano E, Guerra C, Santos E, Barbacid M. 2010. Genetic analysis of Ras signalling pathways in cell proliferation, migration and survival. EMBO I 29: 10911104.

Drosten M, Simon-Carrasco L, Hernandez-Porras I, Lechuga CG, Blasco MT, Jacob HK, Fabbiano S, Potenza N, Bustelo XR, Guerra C, et al. 2017. H-Ras and K-Ras oncoproteins induce different tumor spectra when driven by the same regulatory sequences. Cancer Res 77: 707-718.

Esteban LM, Vicario-Abejon C, Fernandez-Salguero P, Fernandez-Medarde A, Swaminathan N, Yienger K, Lopez E, Malumbres M, McKay R, Ward JM, et al. 2001. Targeted genomic disruption of $\mathrm{H}$-ras and $\mathrm{N}$-ras, individually or in combination, reveals the dispensability of both loci for mouse growth and development. Mol Cell Biol 21: 14441452.

Factor DC, Corradin O, Zentner GE, Saiakhova A, Song L, Chenoweth JG, McKay RD, Crawford GE, Scacheri PC, Tesar PJ. 2014. Epigenomic comparison reveals activation of 'seed' enhancers during transition from naive to primed pluripotency. Cell Stem Cell 14: 854-863.

Faddah DA, Wang H, Cheng AW, Katz Y, Buganim Y, Jaenisch R. 2013. Single-cell analysis reveals that expression of nanog is biallelic and equally variable as that of other pluripotency factors in mouse ESCs. Cell Stem Cell 13: 23-29.

Galonska C, Ziller MJ, Karnik R, Meissner A. 2015. Ground state conditions induce rapid reorganization of core pluripotency factor binding before global epigenetic reprogramming. Cell Stem Cell 17: 462-470.

Heintzman ND, Stuart RK, Hon G, Fu Y, Ching CW, Hawkins RD, Barrera LO, Van Calcar S, Qu C, Ching KA, et al. 2007. Distinct and predictive chromatin signatures of transcriptional promoters and enhancers in the human genome. Nat Genet 39: 311-318.

Hnisz D, Abraham BI, Lee TI, Lau A, Saint-Andre V, Sigova AA, Hoke HA, Young RA. 2013. Super-enhancers in the control of cell identity and disease. Cell 155: 934-947.

Huang FW, Mosquera JM, Garofalo A, Oh C, Baco M, Amin-Mansour A, Rabasha B, Bahl S, Mullane SA, Robinson BD, et al. 2017. Exome sequencing of African-American prostate cancer reveals loss-of-function ERF mutations. Cancer Discov 7: 973-983.

Kunath T, Saba-El-Leil MK, Almousailleakh M, Wray J, Meloche S, Smith A. 2007. FGF stimulation of the Erk1/2 signalling cascade triggers transition of pluripotent embryonic stem cells from self-renewal to lineage commitment. Development 134: 2895-2902.

Landeira D, Sauer S, Poot R, Dvorkina M, Mazzarella L, Jorgensen HF, Pereira CF, Leleu M, Piccolo FM, Spivakov M, et al. 2010. Jarid2 is a PRC2 component in embryonic stem cells required for multi-lineage differentiation and recruitment of PRC1 and RNA Polymerase II to developmental regulators. Nat Cell Biol 12: 618-624.

Le Gallic L, Sgouras D, Beal G Jr, Mavrothalassitis G. 1999. Transcriptional repressor ERF is a Ras/mitogen-activated protein kinase target that regulates cellular proliferation. Mol Cell Biol 19: 4121-4133.

Le Gallic L, Virgilio L, Cohen P, Biteau B, Mavrothalassitis G. 2004. ERF nuclear shuttling, a continuous monitor of Erk activity that links it to cell cycle progression. Mol Cell Biol 24: 1206-1218.

Li J, Wang G, Wang C, Zhao Y, Zhang H, Tan Z, Song Z, Ding M, Deng H. 2007. MEK/ERK signaling contributes to the maintenance of human embryonic stem cell self-renewal. Differentiation 75: 299-307.

Lopez-Contreras AJ, Gutierrez-Martinez P, Specks J, Rodrigo-Perez S, Fernandez-Capetillo O. 2012. An extra allele of Chk1 limits oncogene-induced replicative stress and promotes transformation. J Exp Med 209: 455-461.

Lu CW, Yabuuchi A, Chen L, Viswanathan S, Kim K, Daley GQ. 2008. Ras-MAPK signaling promotes trophectoderm formation from embryonic stem cells and mouse embryos. Nat Genet 40: 921-926.

Marks H, Kalkan T, Menafra R, Denissov S, Jones K, Hofemeister H, Nichols J, Kranz A, Stewart AF, Smith A, et al. 2012. The transcriptional and epigenomic foundations of ground state pluripotency. Cell 149: 590-604.

McLean CY, Bristor D, Hiller M, Clarke SL, Schaar BT, Lowe CB, Wenger AM, Bejerano G. 2010. GREAT improves functional 
interpretation of cis-regulatory regions. Nat Biotechnol 28: 495-501.

Muzumdar MD, Chen PY, Dorans KJ, Chung KM, Bhutkar A, Hong E, Noll EM, Sprick MR, Trumpp A, Jacks T. 2017. Survival of pancreatic cancer cells lacking KRAS function. Nat Commun 8: 1090.

Oeda S, Hayashi Y, Chan T, Takasato M, Aihara Y, Okabayashi K, Ohnuma K, Asashima M. 2013. Induction of intermediate mesoderm by retinoic acid receptor signaling from differentiating mouse embryonic stem cells. Int J Dev Biol 57: 383389.

Potenza N, Vecchione C, Notte A, De Rienzo A, Rosica A, Bauer L, Affuso A, De Felice M, Russo T, Poulet R, et al. 2005. Replacement of K-Ras with $\mathrm{H}$-Ras supports normal embryonic development despite inducing cardiovascular pathology in adult mice. EMBO Rep 6: 432-437.

Pylayeva-Gupta Y, Grabocka E, Bar-Sagi D. 2011. RAS oncogenes: weaving a tumorigenic web. Nat Rev Cancer 11: 761-774.

Samatar AA, Poulikakos PI. 2014. Targeting RAS-ERK signalling in cancer: promises and challenges. Nat Rev Drug Discov 13: 928-942.

Sgouras DN, Athanasiou MA, Beal GJ Jr, Fisher RJ, Blair DG, Mavrothalassitis GJ. 1995. ERF: an ETS domain protein with strong transcriptional repressor activity, can suppress ets-associated tumorigenesis and is regulated by phosphorylation during cell cycle and mitogenic stimulation. EMBO I 14: 4781-4793.

Sizemore GM, Pitarresi JR, Balakrishnan S, Ostrowski MC. 2017. The ETS family of oncogenic transcription factors in solid tumours. Nat Rev Cancer 17: 337-351.

Smith AG, Heath JK, Donaldson DD, Wong GG, Moreau J, Stahl M, Rogers D. 1988. Inhibition of pluripotential embryonic stem cell differentiation by purified polypeptides. Nature 336: 688-690.

Takahashi K, Mitsui K, Yamanaka S. 2003. Role of ERas in promoting tumour-like properties in mouse embryonic stem cells. Nature 423: 541-545.

Ter Huurne M, Chappell J, Dalton S, Stunnenberg HG. 2017. Distinct cell-cycle control in two different states of mouse pluripotency. Cell Stem Cell 21: 449-455 e444.

Twigg SR, Vorgia E, McGowan SJ, Peraki I, Fenwick AL, Sharma VP, Allegra M, Zaragkoulias A, Sadighi Akha E, Knight SJ, et al. 2013. Reduced dosage of ERF causes complex craniosynostosis in humans and mice and links ERK1/2 signaling to regulation of osteogenesis. Nat Genet 45: $308-313$.

Verykokakis M, Papadaki C, Vorgia E, Le Gallic L, Mavrothalassitis G. 2007. The RAS-dependent ERF control of cell proliferation and differentiation is mediated by c-Myc repression. I Biol Chem 282: 30285-30294.

Williams RL, Hilton DJ, Pease S, Willson TA, Stewart CL, Gearing DP, Wagner EF, Metcalf D, Nicola NA, Gough NM. 1988. Myeloid leukaemia inhibitory factor maintains the developmental potential of embryonic stem cells. Nature 336: 684-687.

Yang H, Schramek D, Adam RC, Keyes BE, Wang P, Zheng D, Fuchs E. 2015. ETS family transcriptional regulators drive chromatin dynamics and malignancy in squamous cell carcinomas. Elife 4: e10870.

Ying QL, Nichols J, Chambers I, Smith A. 2003. BMP induction of Id proteins suppresses differentiation and sustains embryonic stem cell self-renewal in collaboration with STAT3. Cell 115: 281-292.

Ying QL, Wray J, Nichols J, Batlle-Morera L, Doble B, Woodgett J, Cohen P, Smith A. 2008. The ground state of embryonic stem cell self-renewal. Nature 453: 519-523. 


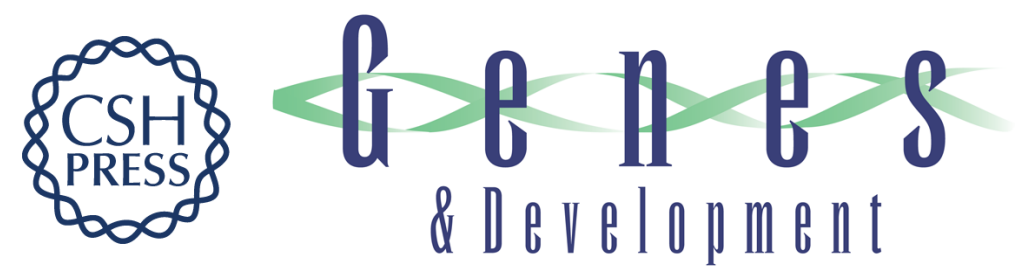

\section{ERF deletion rescues RAS deficiency in mouse embryonic stem cells}

Cristina Mayor-Ruiz, Teresa Olbrich, Matthias Drosten, et al.

Genes Dev. 2018, 32: originally published online April 12, 2018

Access the most recent version at doi:10.1101/gad.310086.117

\section{Supplemental Material \\ References \\ Creative \\ Commons \\ License}

Email Alerting

Service
http://genesdev.cshlp.org/content/suppl/2018/04/12/gad.310086.117.DC1

This article cites 43 articles, 12 of which can be accessed free at: http://genesdev.cshlp.org/content/32/7-8/568.full.html\#ref-list-1

This article is distributed exclusively by Cold Spring Harbor Laboratory Press for the first six months after the full-issue publication date (see

http://genesdev.cshlp.org/site/misc/terms.xhtml). After six months, it is available under a Creative Commons License (Attribution-NonCommercial 4.0 International), as described at http://creativecommons.org/licenses/by-nc/4.0/.

Receive free email alerts when new articles cite this article - sign up in the box at the top right corner of the article or click here.

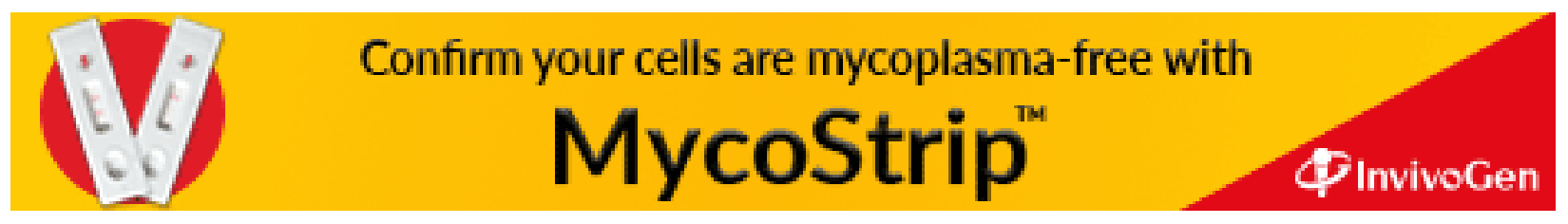

